\title{
LA SUSTITUCIÓN DE TRABAJADORES POR ROBOTS. LA FRONTERA ENTRE LA LIBERTAD DE EMPRESA Y EL DERECHO AL TRABAJO EN LA ERA DIGITAL
}

\author{
Rosa Ma Rodríguez Martín-Retortillo \\ Profesora Ayudante Doctora. \\ Universidad de Vigo
}

\section{Resumen}

En este artículo se plantean los desafíos que se le presentan al Derecho del Trabajo en la era digital, con un enfoque reflexivo, que aborda tanto la dimensión empresarial como la del trabajador. Tras la reciente Sentencia del Juzgado de lo Social no 10 de Las Palmas de Gran Canaria de 23 de septiembre de 2019, que califica como improcedente el despido de una trabajadora tras sustituirla por un robot, se abre el debate acerca de los límites de la libertad de empresa frente al derecho al trabajo del empleado en el siglo XXI. Con ello se pretenden revisar cuáles son esas fronteras en este nuevo contexto tecnológico de avance imparable.

Palabras clave

Libertad, trabajo, era digital, robotización, retos. 


\section{El trabajador frente a la máquina. un debate que cobra actualidad}

El cara a cara entre el ser humano y la máquina no es nuevo. En la Inglaterra del S.XIX, con la Primera Revolución Industrial, se generó una fuerte respuesta por parte de los trabajadores ante la irrupción de modernos telares en las fábricas textiles. Su reacción, fruto de un creciente descontento ante la paulatina reducción de su trabajo por la introducción de nuevas máquinas, dio lugar a un movimiento contrario a estas, conocido como ludismo. El nombre, según se ha señalado por parte de la doctrina, podría derivar de Ned Ludd, quien sería el primer trabajador que rompió un telar como respuesta al fenómeno industrializador ${ }^{1}$. El movimiento, que tuvo una vida de pocos años -se sucedió entre 1811 y $1816^{2}$ - , contó entre sus defensores con exponentes tan renombrados como el poeta Lord Byron ${ }^{3}$.

Sin embargo, la conflictividad que puede generar hoy en día la Cuarta Revolución Industrial dista mucho de aquel fenómeno. El pulso entre ser humano y máquina se juega en los Tribunales, como se verá más adelante, a través de la casuística que ejemplifica este fenómeno.

Asimismo, hoy en día se ha producido un cambio radical en el planteamiento de las relaciones de trabajo, debido a los avances de las nuevas tecnologías y de la inteligencia artificial. Desde el punto de vista empresarial, dicha irrupción se ha recibido con agrado, dado que la automatización de los procesos de producción conlleva, principalmente, una considerable reducción de costes, en la me- dida en que requiere menos personal y acelera la obtención de resultados. Sin embargo, en lo que respecta a la persona trabajadora, la implementación de estos medios tecnológicos es un arma de doble filo. En su vertiente positiva contribuye a la prestación del servicio de una manera más eficiente -piénsese, a modo de ejemplo, en la labor de los cirujanos, que cuentan con tecnología de máxima precisión para facilitar la ejecución de las operaciones quirúrgicas-. Pero también tiene una vertiente negativa, si se valora desde la perspectiva del rendimiento -esto es, tiempo de ejecución y de respuesta de la tarea del humano frente a la máquina-, y, sobre todo, por su posible colisión con los derechos laborales.

El conflicto no es baladí, especialmente si se tiene en cuenta que las máquinas pueden llegar a vaciar de contenido la prestación de trabajo y, por tanto, desembocar en la extinción de la relación laboral. Con ello, el conflicto entre el derecho a la libertad de empresa y el derecho al trabajo recobra una innegable actualidad.

Por otro lado, también se está generando un intenso debate en clave de futuro. El eje de la discusión gira en torno a si la digitalización implicará una reducción considerable del empleo, o simplemente supondrá un cambio en la forma de trabajar. En este sentido, la Organización para la Cooperación y el Desarrollo Económicos (en lo sucesivo, OCDE) ha publicado recientemente un breve informe sobre el futuro del trabajo en España ${ }^{4}$, en el que ha destacado que en nuestro país «existen más puestos de trabajo con alto riesgo de automatización que la media OCDE: 21.7\% de

\footnotetext{
${ }^{1}$ Al respecto, véase J. CHAVES PALACIOS, "Desarrollo tecnológico en la primera revolución industrial", Norba, Revista de Historia, Vol. 17 (2004, pág. 99). También sobre el tema, F. MANUEL, K. ROBINS, y F. WEBSTER, Máquina maldita: contribuciones para una historia del ludismo, Alikornio (2002); y D.F. NOBLE, Una versión diferente del progreso: en defensa del luddismo, Alikornio (2000). No obstante, no se ha acreditado totalmente su existencia. En este sentido, A. DELGADO, La sociedad hiperdigital. Las 10 fuerzas que cambiarán nuestras vidas, Libros de Cabecera (Barcelona, 2018).

2 BENEDIKT FREY, C., y OSBORNE, M.A., «The future of employment: how susceptible are jobs to computerization» (2013), Technological Forecasting and Social Change, núm.114 (January 2013), pág. 7.

${ }^{3}$ A. ALONSO; I. ARZOZ, Carta al Homo ciberneticus, Edaf-Ensayo (2003), pág. 205.

${ }^{4}$ Localizable en https://www.oecd.org/spain/Employment-Outlook-Spain-ES.pdf.
} 
los trabajadores españoles ocupan un puesto de trabajo con alto riesgo de automatización (...) [y] además, otro $30.2 \%$ ocupa puestos con riesgo de reestructuración de entre 50 y $70 \% »^{5}$. Ello no implica su desaparición, pero sin duda supone un replanteamiento de los esquemas tradicionales. Ante esta situación, conviene reflexionar sobre los retos tiene por delante el Derecho del Trabajo y cómo afrontarlos, lo que se abordará al final de este trabajo.

\section{La era digital y el nuevo plantea- miento de las relaciones laborales. La robotización del empleo}

\section{Libertad de emrpesa versus derecho al trabajo}

Desde el punto de vista jurídico, la entrada de los últimos avances tecnológicos en el ámbito laboral está siendo muy controvertida, en la medida en que se suscita un delicado conflicto, que afecta, por una parte, a libertad de empresa, y por otra, al derecho al trabajo. El artículo 38 de la Constitución "reconoce la libertad de empresa en el marco de la economía de mercado; los poderes públicos garantizan y protegen su ejercicio y la defensa de la productividad, de acuerdo con las exigencias de la economía general y, en su caso, de la planificación». Por su parte, el art. 35.1 del texto constitucional establece que "todos los españoles tienen el deber de trabajar y el derecho al trabajo, a la libre elección de profesión u oficio, a la promoción a través del trabajo y a una remuneración suficiente para satisfacer sus necesidades y las de su familia, sin que en ningún caso pueda hacerse discriminación por razón de

${ }^{5}$ Ibidem

${ }^{6}$ El apartado 1 señala que "se reconoce el derecho a la propiedad privada..." y en el apartado 2 le atribuye una función social que delimitará su contenido.

${ }^{7}$ R. ALEXY, Teoría de los derechos fundamentales, Centro de Estudios Políticos y Constitucionales, (1993).

${ }^{8}$ STC 223/1980, referencia RTC 1981\22, Aranzadi Westlaw.

${ }^{9}$ Fundamento jurídico octavo. sexo». A partir de aquí, surgen las preguntas, que aunque ya han sido abordadas por los Tribunales, hoy en día adquieren de nuevo protagonismo.

Si se parte del tenor literal del art. 38 de la Constitución, la libertad de empresa se plantea de una forma muy amplia o abierta, en tanto en cuanto es reconocida y se garantiza su protección por parte de los poderes públicos. Además, este precepto, debe cohonestarse con el art. 33, relativo al derecho de propiedad 6 . De ahí se puede extraer que, además de la vertiente de entrada y salida del mercado, la libertad de empresa tiene un componente fundamental, que es la libre organización de los recursos que le son propios. Aquí se encuentra el punto de partida del debate. Lo determinante será identificar qué parte de ese contenido es esencial, y por tanto, persiste tras aplicar el triple juicio de proporcionalidad estudiado por Robert ALEXY', que pasa por los parámetros de necesidad, idoneidad y proporcionalidad en sentido estricto. $Y$ aquí es donde el derecho al trabajo puede operar como límite, pero ¿con qué alcance? Habrá que analizar si, en atención a un objetivo legítimo, el sacrificio de un derecho en favor del otro es razonable y está justificado.

En cuanto al derecho al trabajo, supone poder optar a un puesto de trabajo en condiciones de igualdad, siempre que se reúnan los requisitos de capacitación exigidos para el puesto. En su Sentencia de 3 de julio de $1981^{8}$, el Tribunal Constitucional señaló que "el derecho al trabajo no se agota en la libertad de trabajar; supone también el derecho a un puesto de trabajo y como tal presenta un doble aspecto: individual y colectivo, ambos reconocidos en los arts. 35.1 y 40.1 de nuestra Constitución, respectivamente ${ }^{9}$. 
Así, "en su aspecto individual, se concreta en el igual derecho de todos a un determinado puesto de trabajo si se cumplen los requisitos necesarios de capacitación, y en el derecho a la continuidad o estabilidad en el empleo, es decir, a no ser despedidos si no existe una justa causa» ${ }^{10}$. Y "en su dimensión colectiva el derecho al trabajo implica además un mandato a los poderes públicos para que lleven a cabo una política de pleno empleo, pues en otro caso el ejercicio del derecho al trabajo por una parte de la población lleva consigo la negación de ese mismo derecho para otra parte de la misma» ${ }^{11}$.

Pues bien, conviene destacar que ambos derechos, encuadrados en el Título I de la Constitución, se proclaman -no de manera casual- como exponentes de la libertad económica de la persona, tanto en su vertiente mercantil como laboral. Coexisten y se necesitan, en la medida en que de su interrelación se derivan una serie de derechos y deberes recíprocos para empresario y trabajador. En el marco jurídico-laboral, la organización de los recursos por parte de la empresa se manifiesta de diversas maneras. Entre ellas, en el ius variandi -poder de dirección y control del empleador- (art. 20 ET), que le faculta a gestionar y fiscalizar la actividad laboral, siempre de modo que no vulnere los derechos de los trabajadores. También, en la libertad de contratación, siempre que no concurra ningún tipo de discriminación [art. 4., letra c) ET]. O en la extinción del contrato de trabajo por voluntad del empresario, basada, eso sí, en causas tasadas estipuladas por la ley. Entre estas, aquí interesan las causas objetivas [art. 51.1 para los despidos colectivos, y art. 52, letra c) ET para los individuales], que son las conocidas causas ETOP (económicas, técnicas, or- ganizativas y de producción). Y es en este punto donde la irrupción de la digitalización plantea un dilema: el de amortizar el puesto de trabajo como consecuencia de su sustitución del trabajador por un robot. ¿Es una razón técnica? ¿obedece a un fin legítimo que merezca el sacrificio del trabajo del empleado? Esto es lo que se tratará de aclara en este trabajo a través del análisis de algunas Sentencias sobre la materia.

\section{La sustitución de trabajadores por "robots" o "bots"}

\subsection{Concepto}

Mucho se ha dicho sobre la robótica y la inteligencia artificial, pero ¿cómo definirlas? Se ha tratado de dar una aproximación al concepto por parte de diversas fuentes, entre ellas, la Comisión Europea. En su Comunicación, titulada "Inteligencia Artificial para Europa", de 25 de abril de $2018^{12}$ señaló que "el término "inteligencia artificial" (IA) se aplica a los sistemas que manifiestan un comportamiento inteligente, pues son capaces de analizar su entorno y pasar a la acción -con cierto grado de autonomía- con el fin de alcanzar objetivos específicos".

Por otro lado, en cuanto a la robótica, se ha indicado que "el término "Robot" fue acuñado por el escritor checoslovaco Karel Kapek, fallecido en 1938, que adquirió fama mundial con su obra R.U.R, en la que presenta al obrero moderno como un esclavo mecánico, es allí donde justamente emplea la palabra Robot, tomada del eslavo Robota, que significa trabajo» ${ }^{13}$. En cuanto al alcance del concepto, el Parlamento Europeo apuntó, en su Resolución de 16 de febrero de

\footnotetext{
11 Ibidem.

12 Localizable en https://ec.europa.eu/transparency/regdoc/rep/1/2018/ES/COM-2018-237-F1-ES-MAIN-PART-1.PDF

${ }^{13}$ Guía técnica de Seguridad en robótica, Cepyme (Aragón, 2006), pág. 7. Localizable en https://higieneyseguridadlaboralcvs. files.wordpress.com/2012/09/guc3ada-tc3a9cnica-de-seguridad-en-robc3b3tica.pdf
} 
2017 , que «la autonomía de un robot puede definirse como la capacidad de tomar decisiones y aplicarlas en el mundo exterior, con independencia de todo control o influencia externos; que esa autonomía es puramente tecnológica y que será mayor cuanto mayor sea el grado de sofisticación con que se haya diseñado el robot para interactuar con su entorno».

En consecuencia, dentro del concepto "robot" o "robótica", se pueden incluir realidades muy diversas, que sobre todo, proponen retos para la sociedad del futuro. Esta Revolución Digital supone el replanteamiento de algunos puestos de trabajo concebidos de manera tradicional, que, con la incorporación de estos elementos tecnológicos, van a variar en su forma de ejecución. Ya se ha apuntado algo acerca de la frontera entre libertad de empresa y derecho al trabajo, y cómo esta disputa puede dar lugar a la amortización de puestos de trabajo. Pero también, en clave de futuro, se plantean desafíos en la nueva economía. Piénsese en empresas de transporte como Uber, Cabify, o las dedicadas al reparto a domicilio, como Glovo o Deliveroo. Hoy en día, se suceden las resoluciones de los Tribunales que tratan de identificar la naturaleza de la prestación llevada a cabo por esas personas, sin una solución pacífica al respecto. Pero hay un eje común: la movilidad. En este sentido, ya se está hablando del transporte autónomo, aunque todavía no haya vehículos comercializados con esas características. En este contexto se puede destacar la instrucción 15/V113, en relación con la Autorización de pruebas o ensayos de investigación realizados con vehículos de conducción automatizada en vías abiertas al tráfico en general ${ }^{15}$. Asimismo, destaca la Resolución de 15 de enero de 2019, del Parlamento Europeo, sobre la conducción autónoma en los transportes europeos (2018/2089(INI)), con reco- mendaciones para avanzar en la regulación de en esta materia. Habrá que ver cómo se va desarrollando esta tecnología, pero es positiva saber que la Unión Europea es consciente de estas nuevas realidades y la necesidad de contar con un marco regulador que las aborde. Quizá cuando esté más desarrollada esta tecnología, el dilema sobre la naturaleza de la prestación esté superado, y los vehículos sean lo suficientemente autónomos como para actuar de forma plenamente independiente de la mano humana.

Otro caso podría ser el de los drones totalmente autónomos, con muchas posibilidades en el ámbito del transporte de pequeñas mercancías o en el campo de la prevención de riesgos laborales. Este tipo de drones no necesitan ser pilotados, y aunque pueden traer consigo muchas ventajas (a la hora de evitar accidentes de trabajo en el ámbito laboral, o contribuir a agilizar el transporte de pequeños paquetes), también atañen riesgos. Por no decir el replanteamiento que implicarían en los perfiles de ciertas profesiones.

Actualmente existe regulación para las aeronaves pilotadas por control remoto (RPA), en virtud del Real Decreto 1036/2017, de 15 de diciembre, por el que se regula la utilización civil de las aeronaves pilotadas por control remoto, y se modifican el Real Decreto 552/2014, de 27 de junio, por el que se desarrolla el Reglamento del aire $y$ disposiciones operativas comunes para los servicios y procedimientos de navegación aérea y el Real Decreto 57/2002, de 18 de enero, por el que se aprueba el Reglamento de Circulación Aérea. Como señala su Exposición de motivos, la norma «establece el marco jurídico definitivo aplicable a la utilización civil de las aeronaves pilotadas por control remoto (RPA) no sujetas a la normativa de la Unión Europea», por lo que el escenario que

${ }^{15}$ 2015. Ministerio del Interior. 
aquí se plantea en relación a los drones no pilotados, aún es un reto.

\subsection{Jurisprudencia de interés}

Como se ha anticipado, la digitalización del mercado laboral plantea nuevas incertidumbres en cuanto a su coexistencia con el trabajo manual. Uno de los puntos más controvertidos viene dado por la amortización de puestos de trabajo como consecuencia de la sustitución de trabajadores por robots. En este sentido, es muy significativa una Sentencia reciente del Juzgado de lo Social $n^{\circ} 10$ de Las Palmas de Gran Canaria, de 23 de septiembre de $2019^{16}$. Esta resolución se pronuncia sobre el despido de una trabajadora sustituida por un robot. No fue la única despedida por la empresa, sino que en la misma fecha fue despedido otro trabajador, y un tercero fue prejubilado. Según figura en los hechos probados, se trata de una trabajadora que prestaba servicios como administrativa (en concreto, oficial de contabilidad) en un hotel en Gran Canaria con una antigüedad de 13 años. La empresa utiliza un Programa de Gestión Hotelera (PMS360) con una serie de funciones básicas, que realizaba desde las 17:15 hasta 06:00, y los festivos y fines de semana las 24 horas. Parte de dichas funciones las ejecutaba el nuevo robot o, mejor llamado, bot de gestión.

En un momento dado, la trabajadora remitió la relación de las tareas que desarrollaba y el tiempo que dedicaba a cada una, y posteriormente recibió la carta de despido por causas productivas, técnicas y organizativas, de conformidad con el art. 52, c) del Estatuto de los Trabajadores. Ante estos hechos, la trabajadora decidió demandar a la empresa, en el entendimiento de que su despi- do era improcedente.

El razonamiento de la Sentencia está muy argumentado y es detallado, pues examina con detenimiento los hechos que llevaron a la empresa a adoptar la decisión extintiva -donde demuestra conocimientos técnicos sobre la materia, más allá de lo estrictamente jurídico-, y analiza exhaustivamente si los mismos encajan en las causas extintivas establecidas por la ley. Así, señala que «la invocación que se hace en la carta de causa productiva no puede ser tal [ya que] las causas productivas afectan al ámbito de los productos o servicios que la empresa pretende colocar en el mercado, y estos productos o servicios, que son de gestión de intermediación turística y de administración, gestión, explotación y gerencia de sociedad dedicadas a la actividad turística no había cambiado al tiempo del despido. No ha surgido un nuevo producto turístico, ni ha desaparecido ninguno. La empresa demandada pretende hacer valer que el mercado turístico está en declive, que los datos y las expectativas son negativos, sin embargo, lo que hace es adelantarse a un futuro que aún no se ha producido. La causa productiva podría afectar a que el producto o servicio que ofrece la empresa, estuviera en franca decadencia, sin embargo, no puede basarse la causa productiva en que dicho producto tiene visos de alcanzar la decadencia en un futuro, incierto y sin proximidad definida» ${ }^{17}$. Asimismo, puntualiza que "todo despido debe basarse en una causa actual, y no en una prevista o previsible de suceder, esto es, no puede basarse en un futurible ${ }^{18}$. Y aquí es lo que ocurre, dado que, a juicio del Tribunal, "a fecha del despido no había un dato objetivo que permitiera justificar un cambio en los productos o servicios que la empresa pretende colocar en el

\footnotetext{
16 JUR\2019\268948, Aranzadi Westlaw.

${ }^{17}$ Fundamento de Derecho cuarto.

18 Ibidem.
} 
mercado, y por ende, justificar el despido» ${ }^{19}$.

A mayores, la empresa alegó causas técnicas y organizativas, al indicar que procedió a la "“robotización de parte de las funciones administrativas que hasta el momento venían siendo desarrolladas directamente por el personal" ». No obstante, la Sentencia señala que "desde un punto de vista puramente técnico, la empresa no ha procedido a la robotización, sino a la automatización a través de software, es decir, no existe "robot" físico alguno que haya asumido tareas, sino que un software dotado de reconocimiento óptico de caracteres, lee determinados documentos, extrae la información y hace con ella aquello para lo que está programado» ${ }^{20}$.

En este caso, la peculiaridad radica en que no se está efectuando un cambio en el medio de producción, sino que el trabajador es sustituido por una máquina. Por tanto, la entidad del cambio no puede ser obviada, no se cambia una máquina por otra, sino que un robot pasa a realizar esas funciones con el único fin de agilizar el proceso productivo y ahorrar costes. Como indica la resolución, «nos hayamos con la aparición de un nuevo elemento, a saber, un software que automatiza lo que antes se realizaba manualmente, prescindiendo por tanto de la necesidad del trabajador humano" ${ }^{21}$. Puntualiza que "las "causas técnicas" parten, entre otros, de un "cambio" en los medios o instrumentos de producción; en el caso de la automatización, más que un cambio entendiendo tal como conversión o modificación de algo en otra cosa - la automatización implica la irrupción de algo nuevo, y no el cambio de algo pasado» 22 . Y concluye que "la automatización mediante 'bots' o 'robots', con la única excusa de reducir costes para aumentar la competitividad, viene a significar los mismo que reducir el Derecho al Trabajo para aumentar la libertad de empresa ${ }^{23}$. Por tanto, ante esos argumentos no hay otro resultado posible que el de declarar el despido improcedente.

Por su parte, aunque no tan recientes, existen pronunciamientos de los Tribunales relativos a la amortización de puestos de trabajo debido a la incorporación de robots. Así, destaca la Sentencia de la Sala de lo Social del Tribunal Superior de Justicia de Madrid de 1 de marzo de 2000²4, relativa al despido de varios trabajadores, una de los cuales llevaba a cabo funciones como Secretaria, afectada por la incorporación de un robot. Entre las funciones de la trabajadora, estaban la de atender "llamadas telefónicas así como pedidos telefónicos (...), la venta y captación de clientes por teléfono, teniendo también la misión de introducir en cada una de las cestas de pedidos $-\mathrm{y}$ hasta la instalación del nuevo robot- los correspondientes albaranes, tarea esta que compartían las seis Secretarias existentes de forma rotativa y en la que se empleaba en torno a tres horas diarias, si bien la demandante desde noviembre de 1997 no entraba en dicho turno y no hacía tales tareas por unas dolencias de espalda y con el permiso de la propia empresa tras diversos en- 
frentamientos por ello» ${ }^{25}$. En la carta de despido dirigida a la trabajadora, la empresa se ampara en razones técnicas, organizativas y de producción, en atención a la dura competencia en el sector. La empresa considera que ante un panorama como el indicado, la innovación tecnológica es la vía más adecuada para que la empresa sea viable y el negocio pueda continuar. Con la implantación de un segundo robot, la empresa pretendía agilizar el tiempo de respuesta a los clientes, pero con ello vaciaba de contenido el trabajo de la empleada, ya que con la incorporación de ese robot, que contaba con un "punto de arranque automático", hacía que los albaranes se activasen de forma automática. En lo que atañe a los demás trabajadores que se vieron afectados por la aparición del segundo robot, se indica que su trabajo habría disminuido en un $40 \%$, por lo que también prescinden de sus servicios.

En la fundamentación jurídica, el Tribunal se remite a su doctrina anterior (Sentencia de 27 de enero de 1998), al señalar que "la causa organizativa o la tecnológica (...) presentan una estructura diádica, de dos elementos, en cuanto la inadecuada organización de los recursos habilita una medida patronal organizativa o tecnológica, en relación derivativa; la dualidad conlleva pues diferenciar el componente volitivo -subjetivo- que supone la adopción de la medida correctora y el componente nolitivo - objetivo- que actúa como presupuesto habilitador y que consiste en la inadecuada organización de los recursos, que mengua la competitividad» ${ }^{26}$. Y por ello destaca que un elemento que hace concluir que la aparición del segundo robot no es motivo suficiente para amortizar esos puestos de trabajo es el hecho de que los trabajadores de la empresa tenían que ha- cer horas extra para poder asumir el cumplimiento de la prestación. En consecuencia, declara los despidos improcedentes.

Otro caso de interés es el resuelto por la Sala de lo Social del Tribunal Superior de Justicia de Madrid, en su Sentencia de 20 abril 200427. Aquí se trata de un despido por causas objetivas, basado en motivos económicos, dadas las pérdidas que había sufrido la empresa. Fueron varios los trabajadores despedidos, y alguno de ellos se vio especialmente afectado por la implantación de un robot para agilizar el trabajo. En concreto, el robot " reduc[ía] la mano de obra en el área de granjeado al existir una disminución de las horas de proceso del $43 \%$ de promedio ${ }^{28}$. En este caso se entendió que, debidamente acreditadas por la empresa las pérdidas económicas, la incorporación del robot contribuía a superar esa situación económica negativa, y ahorrar costes para conseguir sacar a flote el negocio, con lo que los despidos fueron declarados procedentes.

Hasta aquí se han destacado algunos casos en los que el supuesto de hecho giraba en torno a la introducción de medios tecnológicos avanzados que suplieran la actividad del trabajador, con la consiguiente amortización de su puesto. Pero en otras ocasiones, la sustitución de trabajadores por robots puede tener un fin preventivo, en la medida en que permite dotar de mayor seguridad la prestación del servicio que hasta entonces realizaba la persona trabajadora. En este sentido, se puede traer a colación la Sentencia de la Sala de lo Social del Tribunal Superior de Justicia de la Comunidad Valenciana, de 18 de septiembre de $2007^{29}$, en la que un trabajador que prestó servicios veintiocho años como ceramista, estaba ex-

\footnotetext{
${ }^{25}$ Antecedente de hecho segundo.

${ }^{26}$ Fundamento de Derecho cuarto.

${ }^{27}$ AS1200412344, Aranzadi Westlaw.

${ }^{28}$ Antecedente de hecho segundo.

${ }^{29}$ ASI20081244, Aranzadi Westlaw.
} 
puesto, junto con otros compañeros, a polvo de sílice, debido a las características de la cabina de esmaltado en la que realizaba su labor. Finalmente, se le diagnosticó "fibrosis masiva progresiva, equiparable a silicosis de tercer grado, presentado una notable insuficiencia respiratoria» ${ }^{30}$. En una de las cabinas, se sustituyó a la persona por un robot, con lo que pese a que el objeto del caso es la enfermedad profesional del trabajador, se pueden extraer las ventajas que en este contexto ofrece la robótica, dado que se pueden encomendar a los robots, trabajos que tienen un índice de peligrosidad alto para las personas.

\section{Los retos del derecho del trabajo frente a una realidad en constante cambio}

Ante la creciente -e imparable- digitalización del empleo, la OCDE ha señalado que "a pesar de la ansiedad por la destrucción de empleos provocada por la globalización el cambio tecnológico, es improbable que se produzca una fuerte disminución del empleo; en general, la cantidad de puestos de trabajo va en alza y si bien algunos pueden desaparecer (el 14\% tiene un alto riesgo de automatización), otros surgirán» ${ }^{31}$.

Como ya se adelantó, la irrupción de las tecnologías en el entorno laboral ha supuesto un replanteamiento de las relaciones de trabajo tal y como se concebían hasta tiempo recientes ${ }^{32}$. Por ello, conviene detenerse en las dos caras de la moneda, con sus luces y sus sombras. En este sentido es muy ilustrativa la Guía técnica que publicó el Gobierno de Aragón en el año 2006³3, en la que identificaba «nuevos riesgos derivados de la implantación de sistemas automáticos y robotizados». En particular: 1) «mayor nivel de stress derivado de la mayor intensidad y del aumento de la carga de trabajo»; 2) «mayor presión psíquica derivada del requerimiento del ritmo de trabajo del robot, las tareas añadidas $y$, el aumento de las responsabilidades en la toma de decisiones»; 3) «riesgo de accidentes más graves, derivados de las características de las nuevas tecnologías de automatización: riesgos derivados de la maquinaria móvil y del uso de energía eléctrica»; 4) «en áreas de actividad no industriales, como la construcción, la limpieza y mantenimiento de edificios, o la ayuda hospitalaria, riesgos derivados del mal uso o error del robot, relativos a su capacidad móvil, su potencia en el movimiento de objetos, y sus requerimientos energéticos». Como contrapunto, la vertiente positiva que destaca la Guía viene dada por las «mejoras de la salud y la seguridad derivadas de la implantación de sistemas automáticos y robotizados», ya que: 1) "disminuirán el número de accidentes laborales en las empresas con altos niveles de robotización»; 2) «desde el punto de vista físico, la robotización mejora y optimiza las condiciones de trabajo, eliminando riesgos laborales derivados del trabajo en contextos hostiles o con sustancias tóxicas»; y 3) "supresión de trabajos rutinarios y fatigosos".

En este planteamiento de futuro, es inevitable referirse a "las primeras reglas sobre el uso de androides, o al menos las más conocidas, [que] fueron promulgadas (en su relato Runaround) por el científico y divulgador Isaac Asimov» ${ }^{34}$. Estas eran las siguientes: $1^{\text {a }}$ ) «un robot no hará daño a un ser humano o, por inacción, permitirá que un ser humano sufra daño»; $2^{a}$ ) «un robot debe obe-

\footnotetext{
${ }^{30}$ Antecedente de hecho segundo.

${ }^{31}$ Localizable en https://www.oecd.org/spain/Employment-Outlook-Spain-ES.pdf, consultado el 6 de octubre de 2019.

${ }^{32}$ Sobre el tema, también véase BENEDIKT FREY, C., y OSBORNE, M.A., "The future of employment: how susceptible are jobs to computerization» (2013), op. cit.

${ }^{33}$ Guía técnica de Seguridad en robótica, op. cit.

${ }^{34}$ S. RODRíGUEZ BAJÓN, "La era Asimov. Análisis de la propuesta del PE en materia de robótica», La Ley Digital, núm.1392 (2017), pág. 2.
} 
decer las órdenes dadas por los seres humanos, excepto si estas órdenes entrasen en conflicto con la 1. ${ }^{a}$ Ley»; $3^{a}$ ) "un robot debe proteger su propia existencia en la medida en que esta protección no entre en conflicto con la $1 .^{a}$ o la $2 .^{a} "$.

Frente a los peligros y riesgos ya señalados, estas "leyes" podrían servir de punto de partida para plantearse un marco legal detallado y precios, en que se consideren los diferentes ámbitos en los que humanos y robots pueden interactuar. A modo de ejemplo, un aspecto de gran relevancia es el que atañe a las cotizaciones a la Seguridad Social ${ }^{35}$. Aquí se genera cierta controversia, en la medida en que se puede argumentar que la cotización supone que el trabajador se beneficia de la acción protectora del sistema. En cambio, si se habla de robots, no se trataría de un sujeto que generase derechos como los que se desprenden de nuestro actual sistema. La alternativa sería un replanteamiento en la consideración de que con esas cotizaciones podrían contribuir al sostenimiento de nuestro Estado de Bienestar ${ }^{36}$.

En un plano más global, la Organización Internacional del Trabajo (OIT) publicó un informe en enero de $2019^{37}$ con el objeto de reflexionar sobre el futuro del trabajo. Bajo el título “Trabajar para un futuro más prometedor". Entre sus recomendaciones, está la de "encauzar y administrar la tecnología en favor del trabajo decente ${ }^{38}$, y aquí puntualiza que "también que se adopte un enfoque de la inteligencia artificial "bajo control humano" que garantice que las decisiones definitivas que afectan al trabajo sean tomadas por personas ${ }^{39}$.

\section{Conclusión}

Expuesto el tema de la sustitución de trabajadores por robots, es indudable que el papel de los Tribunales en esta materia será determinante. Ante los tímidos e incipientes intentos por parte de Europa de perfilar de algún modo esta materia, la realidad avanza a mucha más velocidad que la norma, y actualmente el panorama económico y laboral plantea muchas incertidumbres que impiden regular algo con garantías reforzadas.

En esa función tan determinante que asume la jurisprudencia, la Sentencia del Juzgado de lo Social $n^{\circ} 10$ de Las Palmas de Gran Canaria trasmite un mensaje tranquilizador, en la medida en que defiende el Derecho del Trabajo desde su esencia. Da a la trabajadora la oportunidad de prestar servicios para su empleador sin que el ahorro de costes por encima de todo sea la razón única y decisiva para extinguir su relación laboral. Por tanto, en los casos que se planteen a futuro, habrá

\footnotetext{
${ }^{35}$ Sobre el tema, véase M.A. GÓMEZ SALGADO, «Robótica, empleo y Seguridad Social: la cotización de los robots para salvar el actual Estado del Bienestar», Revista Internacional y Comparada de Relaciones Laborales y Derecho del Empleo, Vol. 6, núm. 3 (2018). ${ }^{31}$ Localizable en https://www.oecd.org/spain/Employment-Outlook-Spain-ES.pdf, consultado el 6 de octubre de 2019.

${ }^{36}$ En este sentido, la ya citada Resolución del Parlamento Europeo, de 16 de febrero de 2017, con recomendaciones destinadas a la Comisión sobre normas de Derecho civil sobre robótica (2015/2103(INL)), señala que "el desarrollo de la robótica y la inteligencia artificial puede dar lugar a que los robots asuman gran parte del trabajo que ahora realizan los seres humanos sin que puedan reemplazarse por completo los empleos perdidos, cuestión esta que genera interrogantes sobre el futuro del empleo y la viabilidad de los sistemas de seguridad y bienestar sociales y sobre la insuficiencia continuada de las cotizaciones para los regímenes de jubilación, en caso de que se mantenga la actual base imponible, lo que podría acarrear una mayor desigualdad en la distribución de la riqueza y el poder, mientras que, en el marco de la financiación del apoyo y reciclaje profesional para desempleados cuyos puestos de trabajo se hayan reducido o eliminado, deberá estudiarse la posibilidad de someter a impuesto el trabajo ejecutado por robots o exigir un gravamen por el uso y mantenimiento de cada robot, a fin de mantener la cohesión social y la prosperidad».

${ }^{37}$ De fecha 22 de enero de 2019. Resumen localizable en https://www.ilo.org/wcmsp5/groups/public/---dgreports/---cabinet/ documents/publication/wcms_662541.pdf

38 Pág. 4.

39 Ibidem.
} 


\section{LA SUSTITUCIÓN DE TRABAJADORES POR ROBOTS. LA FRONTERA ENTRE LA LIBERTAD DE EMPRESAY EL DERECHO AL TRABAJO EN LA ERA DIGITAL \\ Rosa $M^{a}$ Rodriguez Martín-Retortillo}

que analizar si concurren las causas tasadas por la ley de un modo estricto. El hecho de que en ocasiones ciertas causas extintivas estén formuladas por la ley de una manera demasiado abstracta, no da carta de naturaleza al empresario para tratar de subsumir en las mismas situaciones que no encajan, bajo el pretexto del ahorro de costes.

El enfoque en cuanto a la coexistencia de máquina y ser humano debe ser integrador, como indica la OIT en su informe de enero de 2019. EI avance es imparable, pero con control humano.

Sin duda, hoy cobra más actualidad que nunca la frase de Elbert Hubbard, "una máquina puede hacer el trabajo de 50 hombres corrientes, pero no existe ninguna máquina que pueda hacer el trabajo de un hombre extraordinario».

\section{Bibliografía}

ALEXY, R., Teoría de los derechos fundamentales, Centro de Estudios Políticos y Constitucionales, (1993) ALONSO; ARZOZ, I., Carta al Homo ciberneticus, Edaf-Ensayo (2003) BENEDIKT FREY, C., y OSBORNE, M.A., «The future of employment: how susceptible are jobs to computerization» (2013), Technological Forecasting and Social Change, núm.114 (January 2013).

CHAVES PALACIOS, J., “Desarrollo tecnológico en la primera revolución industrial”, Norba, Revista de Historia, Vol. 17 (2004)

DELGADO, A., La sociedad hiperdigital. Las 10 fuerzas que cambiarán nuestras vidas, Libros de Cabecera (Barcelona, 2018)

GÓMEZ SALGADO, M.A., «Robótica, empleo y Seguridad Social: la cotización de los robots para salvar el actual Estado del Bienestar», Revista Internacional y Comparada de Relaciones Laborales y Derecho del Empleo, Vol. 6, núm. 3 (2018).

MANUEL, F., ROBINS, K. y WEBSTER, F., Máquina maldita: contribuciones para una historia del ludismo, Alikornio (2002)

NOBLE, D.F., Una versión diferente del progreso: en defensa del luddismo, Alikornio (2000)

RODRÍGUEZ BAJÓN, S., «La era Asimov. Análisis de la propuesta del PE en materia de robótica», La Ley Digital, núm.1392 (2017) 
LA SUSTITUCIÓN DE TRABAJADORES POR ROBOTS. LA FRONTERA ENTRE LA LIBERTAD DE EMPRESAY EL DERECHO AL TRABAJO EN LA ERA DIGITAL

Rosa $M^{\text {a }}$ Rodríguez Martin-Retortillo 